\title{
Compact Modeling of Multi-Domain Ferroelectric FETs: Charge Trapping, Channel Percolation and Nucleation-Growth Domain Dynamics
}

\author{
Y. Xiang, M. Garcia Bardon, B. Kaczer, Md Nur K. Alam, L.-Å. Ragnarsson, K. Kaczmarek, \\ B. Parvais, G. Groeseneken and J. Van Houdt
}

\begin{abstract}
The (doped-)hafnia-based ferroelectric FET (FeFET) is a promising candidate for low-power non-volatile memories and shows potential use as a steep-slope lowpower logic device. This requires accurate modeling of the metal-ferroelectric-insulator-silicon (MFIS) gate stack electrostatics. Here we present a hardware-validated FeFET compact model that resolves three key aspects in the MFIS electrostatics pertaining to a multi-domain ferroelectric (FE) layer: 1) the non-radiative multi-phonon process-based charge trapping, 2) the source-to-drain channel percolation due to spatial non-uniformity of FE domain switching and 3) the nucleation-growth domain reversal dynamics using a phenomenological formalism. The polarization charge is calculated by discretized domain switching in transient under distributed coercive fields. Based on the comparison of the model versus experimental data on $\mathrm{Hf}_{0.5} \mathrm{Zr}_{0.5} \mathrm{O}_{2} \mathrm{n}$ FeFET hardware, we prove that the onset of FE $V_{T H}$ lowering starts with the source-to-drain percolation path formation, when enough FE domains have been flipped up by the gate bias. We further demonstrate that the field-independent domain growth is the fundamental origin of the measured steep subthreshold slope during the downward $I_{D}-V_{G}$ sweep. The model ultimately aims to lay down the groundwork for a unified FeFET compact model for both memory- and logic-oriented applications.
\end{abstract}

Index Terms - ferroelectric FETs (FeFETs), polarization switching, charge trapping, threshold voltage $\left(V_{\mathrm{TH}}\right)$ programming, steep subthreshold slope.

\section{INTRODUCTION}

CVER since its advent, the CMOS-compatible (doped-)hafnia $\mathrm{Z}_{\text {ferroelectric materials [1] have ignited a lasting enthusiasm }}$ in FeFET-based low-power non-volatile memories (NVM) $[2,3]$. More recently, the experimentally observed steep subthreshold slope in FeFETs [4,5] has further inspired its potential use in low-power logic technologies but also sparked heated discussions on its exact physical origin [6-9]. In the scenario of NVM, the memory window (MW) lies in the shift of the $V_{\text {TH }}$ state from high ("erased") to low ("programmed") by gate bias-programming [10], while for steep slope FeFETs (SSFeFETs) the so-called "voltage amplification" [6,7] is

This work is supported by imec's Industrial Affiliation Program (IIAP). Y. Xiang, Md N. K. Alam, K. Kaczmarek, G. Groeseneken and J. Van Houdt are with imec, 3001 Leuven, Belgium and also with KU Leuven, 3001 Leuven, Belgium (correspondence email: yang.xiang@imec.be).

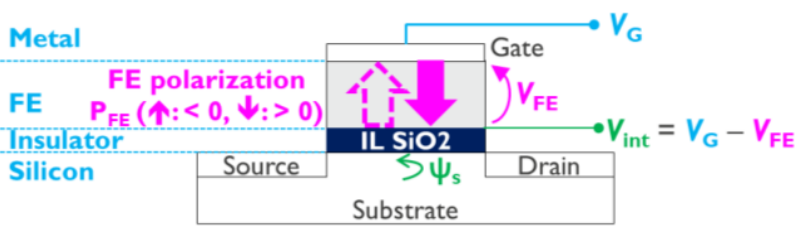

Fig. 1. Schematic of the MFIS electrostatics in a FeFET. "IL" stands for the interfacial layer, and $\psi_{s}$ for surface potential. The FE polarization charge $\mathrm{P}_{\mathrm{FE}}$ in the FE layer alters the voltage drop across it $V_{\mathrm{FE}}$, which accordingly modifies the "internal" gate node $V_{\text {int }}$ above IL and hence the $\psi_{\mathrm{s}}$ at a given $V_{\mathrm{G}}$. This is equivalent to a $V_{\mathrm{TH}}$ shift seen at the $V_{\mathrm{G}}$ node.

believed to concern the FE polarization $\left(\mathrm{P}_{\mathrm{FE}}\right)$-modified surface potential $\psi_{\mathrm{s}}$ change (Fig. 1) that is in effect equivalent to a dynamic $V_{\mathrm{TH}}$ shift. Therefore, from either device physics understanding or technology assessment point of view, it is essential that the MFIS gate stack electrostatics in FeFETs (Fig. 1) be thoroughly investigated and correctly modeled.

In this context, various SPICE FeFET compact models (CMs) have been proposed for either NVMs [11,12] or SSFeFETs $[8,9]$, where the $V_{\mathrm{G}}$-dependent $V_{\mathrm{TH}}$ shift is passed through $V_{\mathrm{FE}}$ (Fig. 1). For all the progress made in existing CMs, there are yet three notable limitations in the $V_{\mathrm{TH}}$ shift modeling. First, the commonly observed charge trapping (CT) $[5,9,13]$ in the polycrystalline (doped-)hafnia FE layer in gate stacks (Fig. 2(a)) is generally overlooked, which is however needed to decouple the trapping-induced positive $\Delta\left|V_{\mathrm{TH}}\right|$ for experimental comparison. Second, the multi-domain (doped-)hafnia FE layer not only introduces a "scalar" coercive field $\left(E_{\mathrm{c}}\right)$ distribution, but also implies a spatial non-uniformity in domain switching $[14,15]$. Existing CMs, failing to address this aspect, would

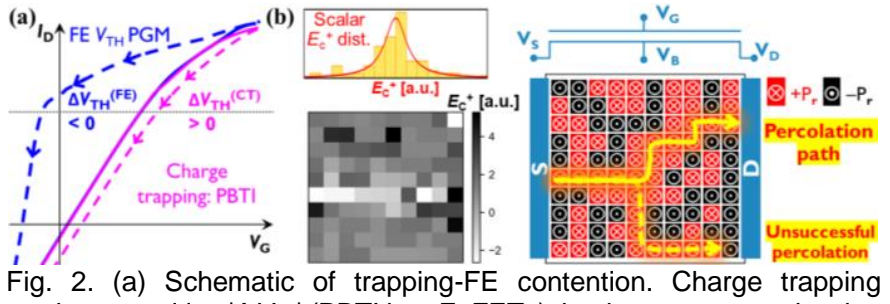
Fig. 2. (a) Schematic of trapping-FE contention. Charge trapping to FE $V_{\mathrm{TH}}$ programming. (b) Schematic of FE $V_{\mathrm{TH}}$ programming in a realworld multi-domain FeFET. The FE domain switching is nonuniformly scattered across the gate area due to the random spatial $E_{\mathrm{c}}$ distribution. Highly inverted source-to-drain percolation paths (solid arrow) beneath the positive domains are thus needed for the FE-induced $V_{\mathrm{TH}}$ reduction to become electrically "visible" (cf. the unsuccessful path in dashes).

M. Garcia Bardon, B. Kaczer and L. $-\AA$ Å. Ragnarsson are with imec, 3001 Leuven, Belgium.

B. Parvais is with imec, 3001 Leuven, Belgium and also with Vrije Universiteit Brussel, 1050 Brussels, Belgium. 


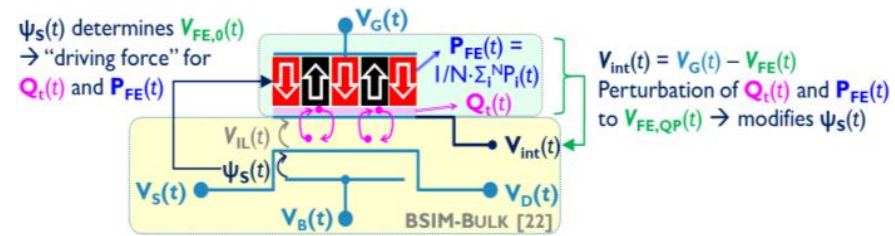

Fig. 3. Schematic of the transient FE-MOS solution in the FeFET CM, where the dual-way (" $\psi_{\mathrm{s}}-$ to- $V_{\mathrm{FE}}$ " and " $V_{\mathrm{FE}}$-to- $V_{\text {int") }}$ ) self-consistent loop is calculated at every time instant [19].

miss the associated channel percolation path formation in order for $V_{\mathrm{TH}}$ shift to effect [16] (Fig. 2(b)). Further missing is a unified modeling approach to reconcile SSFeFETs and NVMoriented FeFETs, whereas in measurements the steep slope behavior has been also observed on NVM-FeFETs [17]. This hints that the steep slope should be derivable from NVMFeFET models based on e.g. domain switching dynamics $[6,8,9]$.

This paper thus reports on a comprehensive FeFET CM aimed at addressing the aforementioned issues on the basis of our recent works $[18,19]$. The paper is organized as follows. In Section II, we introduce the framework of the FeFET CM, including the discretized ferroelectric polarization switching model [20] and the two-state nonradiative multi-phonon (NMP) charge trapping model [21]. In Section III, we elaborate on the percolation-aware FE $V_{\mathrm{TH}}$-programming $\left(V_{\mathrm{TH}}-\mathrm{PGM}\right)$ model and identify the central role of channel percolation in predicting the experimental observations on $\mathrm{Hf}_{0.5} \mathrm{Zr}_{0.5} \mathrm{O}_{2}(\mathrm{HZO}) \mathrm{n}$-FeFETs. In Section IV, we further extend the $V_{\mathrm{TH}}-\mathrm{PGM}$ model with a phenomenological nucleation-growth domain reversal mechanism [6] and confirm the field-independent domain growth as the origin of steep slope $I_{\mathrm{D}}-V_{\mathrm{G}}$ in SSFeFETs. Finally, Section V summarizes the paper.

\section{FeFET Modeling Framework}

\section{A. FeFET CM Framework}

The FeFET model adopts a composite FE-MOS system selfconsistently coupled in transient [19] (Fig. 3). The MOS solution is given by a standard BSIM model [22] that covers the device electrostatics and transport beneath the interfacial layer (IL, normally $\mathrm{SiO}_{x}$ ), with an internal voltage $\left(V_{\text {int }}\right)$ [23] input to the gate node of BSIM. The FE layer simultaneously calculates $V_{\mathrm{FE}}$ via a time-discretized, dual-way feedback loop (Fig. 3) similar to that in $[11,12]$ :

$$
\left\{\begin{array}{c}
V_{F E}(t)=\frac{C_{I L}\left(V_{G}(t)-V_{F B}-\psi_{s}(t)\right)}{C_{I L}+C_{F E}}-\frac{Q_{t}(t)}{C_{I L}+C_{F E}}-\frac{e n v\left(P_{F E}(t)\right)}{C_{I L}+C_{F E}} \\
V_{F E, 0}(t)=C_{I L}\left(V_{G}(t)-V_{F B}-\psi_{s}(t)\right) /\left(C_{I L}+C_{F E}\right) \\
V_{F E, Q}(t)=-Q_{t}(t) /\left(C_{I L}+C_{F E}\right) \\
V_{F E, P}(t)=-e n v\left(P_{F E}(t)\right) /\left(C_{I L}+C_{F E}\right)
\end{array}\right.
$$

Here $\mathrm{C}_{\mathrm{IL}}$ and $\mathrm{C}_{\mathrm{FE}}$ refer to the capacitance of interfacial layer (IL) $\mathrm{SiO}_{\mathrm{x}}$ and the paraelectric high-K component of the FE layer. $\mathrm{Q}_{\mathrm{t}}$ and $e n v\left(\mathrm{P}_{\mathrm{FE}}\right)$ relate to the trapped charge $\mathrm{Q}_{\mathrm{t}}$ and $\mathrm{FE}$ polarization charge $\mathrm{P}_{\mathrm{FE}}$, respectively and will be explained later.

As written in Eqs. (1), $V_{\mathrm{FE}}$ consists of a baseline component $V_{\mathrm{FE}, 0}$ but also two extra components $V_{\mathrm{FE}, \mathrm{Q}}$ and $V_{\mathrm{FE}, \mathrm{P}}$ accounting for $\mathrm{Q}_{\mathrm{t}}$ and $\mathrm{P}_{\mathrm{FE}}$, respectively (Fig. 3). This explicitly embeds voltage balance and charge conservation in the model at each

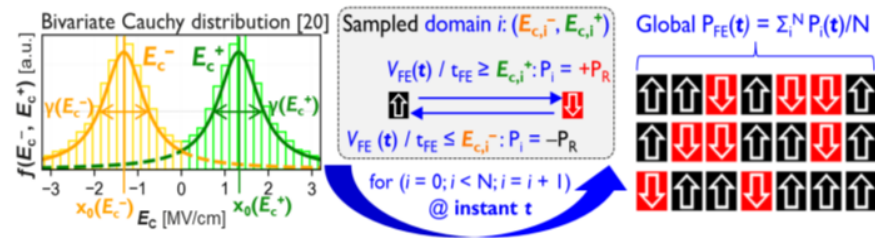

Fig. 4. Schematic of the discretized $P_{F E}$ switching model. The bivariate Cauchy-distributed $f\left(E_{c}^{-}, E_{c}^{+}\right)$is calibrated on metal-FE-metal capacitor measurements using a steady-state Preisach model [20] and used to sample the $N \times$ domains. Here the median $\mathrm{x}_{0}\left(\left|E_{\mathrm{c}}\right|\right)=1.32 \mathrm{MV} / \mathrm{cm}$ and the full width at half maximum $\mathrm{V}\left(\left|E_{\mathrm{c}}\right|\right)=0.57 \mathrm{MV} / \mathrm{cm}$. Note that for domain sampling, the region $f\left(E_{\mathrm{c}}^{-} \geq 0,{E_{\mathrm{c}}}^{+} \leq 0\right)$ is truncated (dashed). The domain polarization $\mathrm{P}_{\mathrm{i}}$ at time $t$ is determined by comparing the sampled coercive fields $\left(E_{\mathrm{c}}^{-},{E_{\mathrm{c}}}^{+}\right)$and the electric field calculated through $V_{\mathrm{FE}}(t)$ in Eqs. (1).

time step $t$ : the $V_{\mathrm{G}}$-dependent $\mathrm{Q}_{\mathrm{t}}$ and $\mathrm{P}_{\mathrm{FE}}$-variation is triggered by the $V_{\mathrm{G}}$-dependent $V_{\mathrm{FE}, 0}$, which already contains the feedback from $\psi_{\mathrm{s}}$. Meanwhile, any $V_{\mathrm{G}}$-dependent perturbation of $\mathrm{Q}_{\mathrm{t}}$ or $\mathrm{P}_{\mathrm{FE}}$ will in turn modify $\psi_{\mathrm{s}}$ through $V_{\mathrm{FE}, \mathrm{Q}}$ and $V_{\mathrm{FE}, \mathrm{P}}$, respectively, such that an eventual convergence is always achieved for the quadruplet $\left(\psi_{\mathrm{s}}, \mathrm{Q}_{\mathrm{t}}, \mathrm{P}_{\mathrm{FE}}, V_{\mathrm{FE}}\right)$.

The self-consistently calculated $V_{\mathrm{FE}}$ will automatically alter $V_{\text {int }}$ and thus translate to an apparent $\Delta V_{\mathrm{TH}}$ seen by the node $V_{\mathrm{G}}$. The $\Delta V_{\mathrm{TH}}$ is derived at iso- $V_{\text {int }}$ condition (equivalently also iso$\psi_{\mathrm{s}}$ ) using Eqs. (1) and it can be conveniently expressed as a function of $\mathrm{Q}_{\mathrm{t}}$ and $\mathrm{P}_{\mathrm{FE}}$ and tracked versus time $t:{ }^{1}$

$$
\left\{\begin{array}{c}
\left.\Delta V_{F E}(t)\right|_{\Delta \psi_{s}=0}=\frac{C_{I L} \Delta V_{G}(t)-\Delta Q_{t}(t)-\Delta e n v\left(P_{F E}(t)\right)}{C_{I L}+C_{F E}} \\
\Delta V_{i n t}(t)=\Delta V_{G}-\Delta V_{F E}=\frac{C_{F E} \Delta V_{G}(t)+\Delta Q_{t}(t)+\Delta e n v\left(P_{F E}(t)\right)}{C_{I L}+C_{F E}} \\
\left.\Delta V_{T H}(t) \triangleq \Delta V_{G}\right|_{\Delta V_{i n t}=0}=-\frac{\Delta Q_{t}(t)}{C_{F E}}-\frac{\Delta e n v\left(P_{F E}(t)\right)}{C_{F E}}
\end{array}\right.
$$

\section{B. Discretized ferroelectric polarization switching}

The global polarization $\mathrm{P}_{\mathrm{FE}}$ in the gate stack is modeled by discretized domain polarization switching in a $\mathrm{FE}$ domain ensemble $[11,12,24]$. This approach does not prescribe a $\mathrm{P}_{\mathrm{FE}^{-}}$ $V_{\mathrm{FE}}$ relation and therefore allows to include the feedback of $\mathrm{P}_{\mathrm{FE}}$ on $V_{\mathrm{FE}}$ per $V_{\mathrm{FE}, \mathrm{P}}$ in Eqs. (1), which is similar to $[11,12]$. The $N$ domains are first instantiated with discrete coercive fields sampled under a calibrated bivariate coercive field distribution $f\left(E_{\mathrm{c}}^{-}, E_{\mathrm{c}}^{+}\right)[20]$. The bistate $\left( \pm \mathrm{P}_{\mathrm{r}}\right)$ domain polarization is then determined at each timestep $t$ via a steady-state instant Preisach switching model (Fig. 4). Finally, the global $\mathrm{P}_{\mathrm{FE}}$ will take the average over all domains at time $t$.

Note that the steady-state Preisach mechanism here uses instant domain dynamics as a first-order approximation. This will be augmented with the nucleation-growth domain dynamics [6] in Section IV, where the exact profile of $f$ will be adjusted accordingly. However, the discretized, "ensemble" domain switching scheme will remain valid. Moreover, the $f\left(E_{\mathrm{c}}^{-}, E_{\mathrm{c}}^{+}\right)$here only concerns the "scalar" $E_{\mathrm{c}}$ distribution without specifying the spatial configuration of the individual $\left(E_{\mathrm{c}}^{-}, E_{\mathrm{c}}^{+}\right)$pairs (Fig. 2(b)). The spatial configuration-related percolation effect will be handled by a dedicated "envelope function" in Section III.

\section{Two-state NMP Charge Trapping Model}



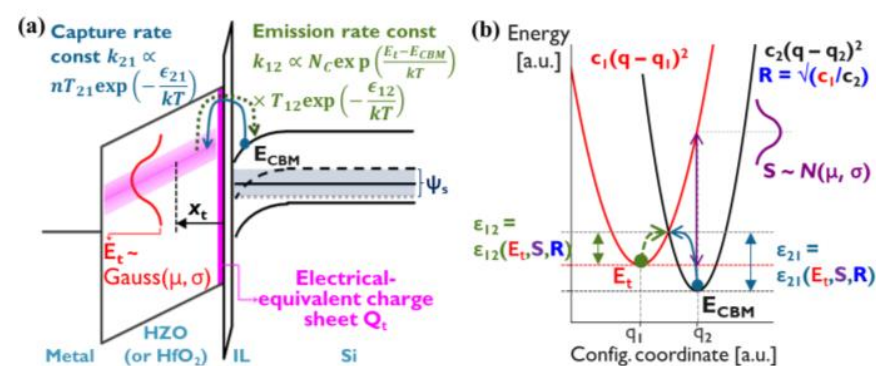

Fig. 5. (a) Schematic of the charge trapping model based on the twostate NMP theory in [21]. The carrier capture and emission rate constants $\left(k_{21}, k_{12}\right)$ are calculated using the trap location $\left(x_{t}\right)$-dependent tunneling coefficients $\left(T_{\mathrm{n} 21}, T_{\mathrm{n} 12}\right)$ and Arrhenius thermal activation barriers $\left(\varepsilon_{12}, \varepsilon_{21}\right)$. (b) Schematic of the Arrhenius thermal barriers $\left(\varepsilon_{12}\right.$, $\left.\varepsilon_{21}\right)$ under the configuration coordinate diagram representation [21]. The $V_{\mathrm{FE}}$-dependence of the rate constants $\left(k_{21}, k_{12}\right)$ effects via the biasdependent trap energy levels $E_{t}$, the field-dependent $\left(T_{n 21}, T_{n 12}\right)$ and the $\mathrm{E}_{\mathrm{t}}$-derived $\left(\varepsilon_{12}, \varepsilon_{21}\right)$ pairs. The capture rate constant $k_{21}$ further depends on the inversion carrier concentration $n$ calculated from BSIM [22].

The bulk charge trapping in the (doped-)hafnia FE layer concerns the dynamic "capture" and "emission" of channel carriers to/from the pre-existing defect sites [21] deep in the (doped-)hafnia bulk (Fig. 5). The vast energetic and spatial distribution of the trapping sites as well as the electron-phonon (e-ph) coupling due to lattice deformation [21] dictate that the "trapping rates" be both broadly distributed and historydependent. Therefore, we apply the two-state NMP model [21] to calculate the individual trap occupancy $\left(p_{\text {occ }}\right)$ across a multidimensional array of traps. The model has been calibrated in [5] using the finite-element solver Comphy [21], with parameters extracted to describe the Gaussian distribution of the trap energy levels $\mathrm{E}_{\mathrm{t}}$ at flat band, the Gaussian distribution of e-ph coupling relaxation energies $S$ and the associated parabola curvature ratio $R$ (Fig. 5).

In the model, the $p_{\text {occ }}$ 's are evaluated at each time instant based on the carrier capture and emission constants $\left(k_{21}, k_{12}\right)$. This is done across all sampled combinations of trap levels and locations $\left(\mathrm{E}_{\mathrm{t}}, x_{\mathrm{t}}\right)$, for all enumerated $S$ values accounting for different $e-p h$ coupling pathways [21]. The equivalent trapped charge sheet $\mathrm{Q}_{\mathrm{t}}$ is then integrated across all $\left(\mathrm{E}_{\mathrm{t}}, x_{\mathrm{t}}\right)$ tuples. Since $\mathrm{Q}_{\mathrm{t}}$ is packed onto the FE/IL boundary, the traps at different locations are assigned different weights depending on $x_{\mathrm{t}}$ [21]. Using the calibrated trap density $\mathrm{N}_{\mathrm{t}}$, the calculations read as follows:

$$
\left\{\begin{array}{c}
\frac{d p_{o c c}(t)}{d t}=-p_{o c c}(t) k_{12}+\left(1-p_{o c c}(t)\right) k_{21} \\
Q_{t}(t)=-e N_{t} t_{F E} \int\left(1-\frac{x_{t}}{t_{F E}}\right) p_{o c c}\left(t, E_{t}, x_{t}\right) d x_{t} d E_{t} \\
\Delta V_{T H}^{(C T)}(t)=-\frac{\Delta Q_{t}(t)}{C_{F E}}
\end{array}\right.
$$

The validation of the "CT-alone" model can be found in our prior works $[18,19]$ on non-FeFET hardware. For FeFET, the major complications are 1) the increase of inversion carrier density $n$ due to switched-up $\mathrm{P}_{\mathrm{FE}}$ and 2) the modification of $V_{\mathrm{FE}}$ through depolarization field [18], both of which will influence the capture/emission rate constants as shown in Fig. 5(a). Thanks to the voltage and charge balance in our CM framework (Section II-A), these two effects are automatically included in the model. Consequently, a trap-aware simulation of the FeFET will not be equal to a "linear superposition" of CT and FE effects. Moreover, our model can be readily extended to include the field cycling-induced fatigue behaviors in FeFETs (e.g.,



(b) Converting resistive network to multi-domain FeFET:



Fig. 6. (a) Schematic of the resistive network (of two sizes: 100/100 and 20/20) simulation per [28]. The fraction of the LR resistors $\varphi_{L R}$ is swept from 0 to 1 while the conductance is simulated using Monte-Carlo. Both the median (symbols) and the spread (shade) in conductance are plotted vs $\varphi_{\mathrm{LR}}$, and $\varphi_{\mathrm{c}}$ denotes the median critical $\varphi_{\mathrm{LR}}$ for percolation. (b) Conversion of resistor network conductance to multi-domain FeFET by comparing $\varphi_{\mathrm{LR}}$ to the normalized $P_{\mathrm{FE}}$. The $G_{\mathrm{norm}}-\varphi_{\mathrm{LR}}$ of resistive network can be then mapped to the FE $V_{T H}$ programming in a FeFET.

reduction of $2 \mathrm{P}_{\mathrm{r}}$ ) [25]. This can be done by considering a higher trap density $\left(\mathrm{N}_{t}\right)$ in the FE layer due to new defects generation (e.g., oxygen vacancies), as well as an increase in gate leakage current due to trap-assisted tunneling [25].

\section{Percolation-Aware Fe $V_{\text {th-Programming Model }}$}

\section{A. Rationale of percolation envelope function for FeFET}

The implication of the spatial $E_{\mathrm{c}}$ non-uniformity in a multidomain (MuD) FE layer can be best illustrated by considering a switching-up process across the spatial " $E_{\mathrm{c}}{ }^{+}$heatmap" in Fig. 2(b). It has been shown by spectroscopic studies [14] that in real-world MuD FE thin films, the flipping up of different FE domains occurs in a successive, scattered manner, with the ones of larger $E_{\mathrm{c}}{ }^{+}$values requiring a higher electric field for flipping to $+\mathrm{P}_{\mathrm{r}}$. Therefore, at a given $V_{\mathrm{FE}}$, if the $+\mathrm{P}_{\mathrm{r}}$ domain population is not large enough, they will not always "cluster" from source to drain to contribute to the highly-inverted "conducting paths" in the channel (e.g., the "unsuccessful" dashed arrow in Fig. 2(b)). It can be then imagined that in a MuD FeFET, the very first source-to-drain percolation path formation would induce a notable $I_{\mathrm{D}}$ increase that can be interpreted as the onset of FEinduced $V_{\mathrm{TH}}$ "programming". Therefore, it is crucial to pinpoint this threshold $+\mathrm{P}_{\mathrm{r}}$ domain population that would define the "percolation limit" $\left(\mathrm{P}_{\mathrm{c}}\right)$ expressing the threshold global polarization charge. Note that, since percolation is inherently a stochastic process [26,27], $\mathrm{P}_{\mathrm{c}}$ is only used to outline the median threshold $\mathrm{P}_{\mathrm{FE}}$ for convenience, while the variability due to percolation stochasticity is beyond the scope of this paper.

In fact, in the dedicated percolation theory developed in statistical physics, the conductance of a resistive network (RN, Fig. 6(a)) has been found to relate to percolation [26-28]. Therefore, if we make an analogy between the $\mathrm{RN}$ and a

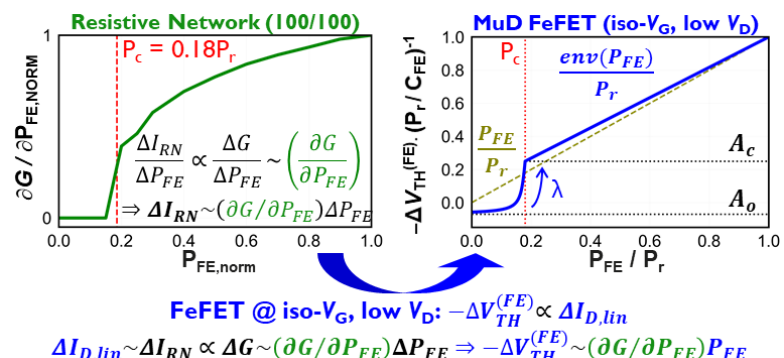

Fig. 7. Construction of the FE $V_{T H} P G M$ expression $\Delta V_{T H}{ }^{(F E)}-P_{F E}$ in FeFET by making an analogy to the $\Delta G-\Delta P_{\mathrm{FE}, \text { norm }}$ in $\mathrm{RN} . \Delta V_{\mathrm{TH}}{ }^{(\mathrm{FE})}$ is wrapped into an envelope function env $\left(\mathrm{P}_{\mathrm{FE}}\right)$ multiplied with pre-factors. Here $\mathrm{P}_{\mathrm{c}}$ depends on transistor $W / L ; \lambda, A_{\mathrm{c}}$ and $A_{\circ}$ are fitting parameters. 
"checkerboard" of $\pm P_{\mathrm{r}}$ FE domains in a MuD FeFET by substituting the high-resistance (HR) resistor for $-\mathrm{P}_{\mathrm{r}}$ and lowresistance (LR) resistor for $+\mathrm{P}_{\mathrm{r}}$, then we can study the percolation-induced $\Delta I_{\mathrm{D}}$ (and $-\Delta V_{\mathrm{TH}}$ ) in a MuD FeFET by looking at the percolation-induced conductance $(G)$ increase in an RN per Fig. 6(b). Indeed, we can recast the fraction of LR resistors $\left(\varphi_{\mathrm{LR}}\right)$ into the normalized global $\mathrm{P}_{\mathrm{FE}}$ in a FeFET and thus link the numerical value of the normalized $\mathrm{P}_{\mathrm{c}}$ to the median percolation threshold $\varphi_{\mathrm{LR}}\left(\varphi_{\mathrm{c}}\right)$. The normalization reads:

$$
\left\{\begin{array}{c}
P_{F E, \text { norm }}=\frac{\#(L R)-\#(H R)}{\#(L R)+\#(H R)}=\varphi_{L R}-\varphi_{H R}=2 \varphi_{L R}-1.0 \\
P_{c, \text { norm }} \triangleq P_{c} / P_{r}=2 \varphi_{c}-1.0
\end{array}\right.
$$

Here we use Monte-Carlo SPICE simulations to profile the $G-\varphi_{\text {LR }}$ relation of square RNs of different sizes (100/100 and 20/20, Fig. 6(a)). The size of RN should not be interpreted as the number of grains in the polycrystalline FE thin film, as different $\pm P_{r}$ sub-regions can co-exist within one grain [29]. By sweeping $\varphi_{\mathrm{LR}}$, we find that an abrupt increase of conductance occurs at a median threshold $\varphi_{\text {LR }}$ of ca. 0.59 , namely $\varphi_{\mathrm{c}} \approx 0.59$. This agrees precisely with theoretical studies on the "site percolation" in infinite square lattices [26-28]. Moreover, the $\mathrm{RN}$-extracted $\varphi_{\mathrm{c}}$ perfectly follows the "percolation universality" rule [27], meaning that it stays invariant with the RN size. This means that, for a given "shape" $(W / L)$ of the FeFET, we can consistently apply the $\varphi_{\mathrm{c}}$ obtained on the RN of that $W / L$ to derive the $\mathrm{P}_{\mathrm{c}}$ in the $\mathrm{FE}$ layer, which in the case of square transistors yields $\mathrm{P}_{\mathrm{c}} \approx 0.18 \mathrm{P}_{\mathrm{r}}$. For higher $W / L$ ratios, the $\mathrm{P}_{\mathrm{c}}$ can be similarly obtained from $\mathrm{RN}$ simulations (e.g., $\mathrm{P}_{\mathrm{c}} \approx 0.06 \mathrm{P}_{\mathrm{r}}$ for $W / L=2$ ) which can be found in our recent work [18].

Furthermore, we can construct the FE PGM-induced $\Delta V_{\mathrm{TH}}$ $\left(\Delta V_{\mathrm{TH}}{ }^{(\mathrm{FE})}\right)$ versus $\mathrm{P}_{\mathrm{FE}}$ by studying the $\partial G / \partial \mathrm{P}_{\mathrm{FE}, \text { norm }}$ extracted from the RN (Fig. 7). This is done by approximating the $\Delta I_{\mathrm{D} \text {,in- }}$ $\mathrm{P}_{\mathrm{FE}}$ in a FeFET at iso- $V_{\mathrm{G}}$ condition to the $\Delta G-\Delta \mathrm{P}_{\mathrm{FE} \text {.norm }}$ in an $\mathrm{RN}$ $\left(\mathrm{P}_{\mathrm{FE}, \text { norm }}\right.$ already converted from $\varphi_{\mathrm{LR}}$ per Eqs. (4)). Since for a given $V_{\mathrm{G}}, \Delta I_{\mathrm{D} \text {,lin }}$ is proportional to $-\Delta V_{\mathrm{TH}}$, the $-\Delta V_{\mathrm{TH}}{ }^{(\mathrm{FE})}$ versus $\mathrm{P}_{\mathrm{FE}}$ can be abstracted into a relation qualitatively similar to $\Delta G-$ $\Delta \mathrm{P}_{\mathrm{FE} . n o r m}$ and cast as an "envelope function" env $\left(\mathrm{P}_{\mathrm{FE}}\right)$ (Fig. 7). The physical interpretation of $\operatorname{env}\left(\mathrm{P}_{\mathrm{FE}}\right)$ is that the FE $V_{\mathrm{TH}} \mathrm{PGM}$ only becomes noticeable when the source-to-drain percolation of $+\mathrm{P}_{\mathrm{r}}$ domains sets in at $\mathrm{P}_{\mathrm{FE}} \gtrsim \mathrm{P}_{\mathrm{c}}$, while for $\mathrm{P}_{\mathrm{FE}} \ll \mathrm{P}_{\mathrm{c}}$ the $+\mathrm{P}_{\mathrm{r}}$ domain population is too small to have the chance of percolation and hence does not contribute to FE $V_{\mathrm{TH}} \mathrm{PGM}$. Since for $\mathrm{P}_{\mathrm{FE}} \ll \mathrm{P}_{\mathrm{c}}$ the channel current is negligible, the $-V_{\mathrm{TH}}{ }^{(\mathrm{FE})}$ value at the "de-percolated" state cannot be deduced from the $\mathrm{RN}$ conductance. For this reason, we leave it as a fitting parameter " $A_{\mathrm{o}}$ " (Fig. 7) that can be subject to the operating history of the FeFET such as the degree of "erasing" (or "reset"). The piecewise $e n v\left(\mathrm{P}_{\mathrm{FE}}\right)$ and the $\Delta V_{\mathrm{TH}}{ }^{(\mathrm{FE})} \mathrm{read}$ :

$$
\left\{\begin{array}{c}
\operatorname{env}\left(P_{F E}\right)=P_{r}\left\{\frac{2\left(A_{c}-A_{o}\right) \operatorname{atan}\left[\lambda\left(P_{F E}-P_{c}\right)\right]}{\pi}+A_{c}\right\}, P_{F E}<P_{c} \\
\operatorname{env}\left(P_{F E}\right)=P_{r}\left[\frac{1-A_{c}}{P_{r}-P_{c}}\left(P_{F E}-P_{c}\right)+A_{c}\right], P_{F E} \geq P_{c} \\
V_{T H}^{(F E)}(t)=-\frac{\Delta \operatorname{env}\left(P_{F E}(t)\right)}{C_{F E}}
\end{array}\right.
$$

Note that the env $\left(\mathrm{P}_{\mathrm{FE}}\right)$ also enters the $V_{\mathrm{FE}}$ expression in Eqs. (1), which not only shifts $V_{\mathrm{TH}}$ but also influences the calculation of $\mathrm{P}_{\mathrm{FE}}$ and $\mathrm{Q}_{\mathrm{t}}$ (Figs. 4 \& 5, respectively). Hence the $V_{\mathrm{FE}}$ should be interpreted as an "electrical equivalent" global potential
TABLE I

Calibrated FE Layer PARAMETERS For ExPerimental PREDiction

\begin{tabular}{c|c|c}
\hline \hline Parameter $^{\mathrm{a}}$ & Median value & Spread \\
\hline Trap type & Acceptor-like & - \\
Trap density $\mathrm{N}_{\mathrm{t}}$ & $2.6 \times 10^{20} \mathrm{~cm}^{-3}$ & - \\
Trap level $\mathrm{E}_{\mathrm{t}}$ at flatband condition ${ }^{\mathrm{b}}$ & $0.83 \mathrm{eV}$ & $0.22 \mathrm{eV}$ \\
Relaxation energy $S$ & $1.9 \mathrm{eV}$ & $1.2 \mathrm{eV}$ \\
Parabola curvature ratio $R$ & 0.32 & - \\
\hline FeFET FE layer thickness $\mathrm{t}_{\mathrm{FE}}$ & $8 \mathrm{~nm}$ & - \\
FE layer dielectric constant $\mathrm{k}_{\mathrm{FE}}$ & 37 & - \\
Saturation polarization $\mathrm{P}_{\mathrm{r}}$ & $22.1 \mu \mathrm{C} / \mathrm{cm}^{2}$ & - \\
Coercive field $\left|E_{\mathrm{c}}\right|$ & $1.32 \mathrm{MV} / \mathrm{cm}$ & $0.57 \mathrm{MV} / \mathrm{cm}$ \\
IL SiO thickness $_{\mathrm{IL}}$ & $0.8 \mathrm{~nm}$ & - \\
\hline \hline${ }^{\mathrm{a}}$ Trap band information per $[5]$, FE parameters per [20]. \\
${ }^{\mathrm{b}}$ Trap level referenced to Si mid-gap at the boundary of IL-SiO $/ \mathrm{Si}$. \\
drop.
\end{tabular}

\section{B. Percolation-based reproduction of experimental data}

Below we discuss the modeling prediction of the measurement results on HZO planar n-FeFET hardware. The devices are fabricated with a replacement-metal-gate planar technology that can be found in [5]. Additional TiN/HZO/TiN metal-FE-metal capacitors (MFMCAPs) are used for FE-HZO thin film characterization [20]. Table I lists the calibrated trap band information in the FE layer in the FeFET gate stack [5] as well as the extracted HZO thin film properties on $100 \mu \mathrm{m} \times 100 \mu \mathrm{m}$ MFMCAPs [20]; the HZO thin film shows typical Preisach-extracted saturation polarization $\left(\mathrm{P}_{\mathrm{r}}\right)$ and coercive field $\left(E_{\mathrm{c}}\right)$ values in literature [8].

1) BTI extended measure-stress-measure characterization

We use the extended measure-stress-measure (eMSM) technique [30] to study the $V_{\mathrm{TH}}$ shift versus voltage pulses of different overdrive amplitude and time (" $V_{\text {ov }}$ " and " $t_{\text {stress }}$ " in Fig. $8(\mathrm{a})$, respectively). The pulses are referenced to the initial $V_{\mathrm{TH}}$ of the device $\left(V_{\mathrm{TH}, 0}\right)$ and the $\Delta V_{\mathrm{TH}}$ is measured at $t_{\text {measure }}$ after a pulse. We assume that the multiple stresses pulses are mutually independent due to the long relaxation time in between (Fig. 8(a)). Clearly, our FeFET CM can reproduce the $\Delta V_{\mathrm{TH}}-V_{\text {ov }}$ trend observed in experiments, which span four decades of different stress times. Here the percolation limit $\mathrm{P}_{\mathrm{c}} \approx 0.18 \mathrm{P}_{\mathrm{r}}$ is used for the square transistor $(W / L=10 \mu \mathrm{m} / 10 \mu \mathrm{m})$, based on our RNbased study in Section III-A. The trap band and FE layer assume the calibrated parameters in Table I.

It turns out that the percolation mechanism in our FeFET model is indispensable for the modeling prediction of the measured $\Delta V_{\mathrm{TH}}-V_{\mathrm{ov}}$ "turnaround" feature (Fig. 9(a)). Such

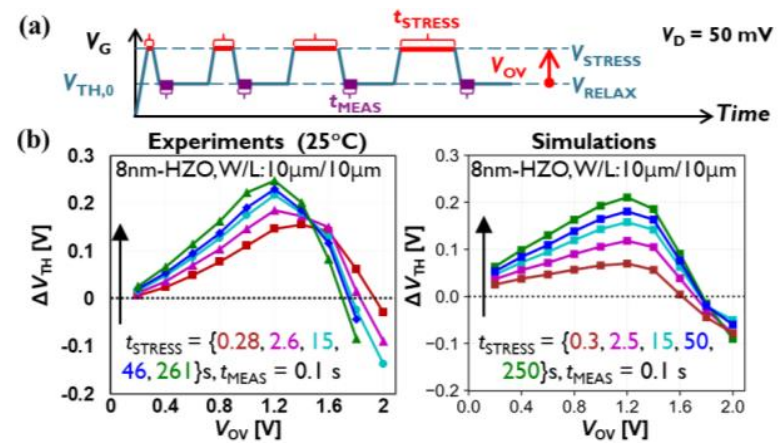

Fig. 8. (a) The applied $V_{G}$ waveform during eMSM characterization. The $V_{\mathrm{TH}}$ shift of the device is measured at $t_{\text {meas }}$ after stress of different length $\left(t_{\text {stress }}\right)$ and amplitude $\left(V_{\text {ov }}\right)$. The pulses are separated by long relaxation times (> $2000 \mathrm{~s}$ ). (b) The comparison between the experimental and simulated $\Delta V_{T H}-V_{\text {ov }}$ in eMSM across four decades of stress times. 

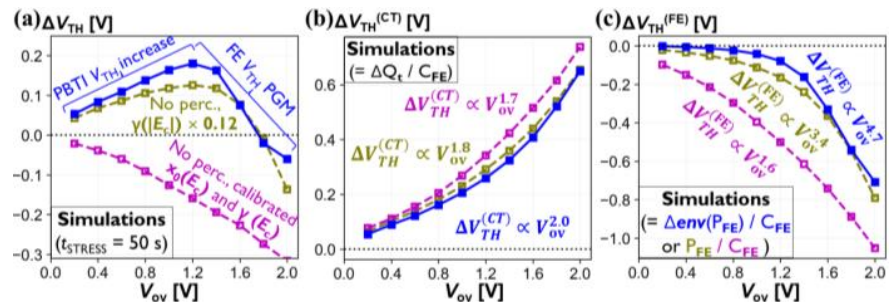

Fig. 9. Visualization of the role of percolation in eMSM modeling. (a): The $\Delta V_{\mathrm{TH}}-V_{\mathrm{ov}}$ "turnaround" in experiments (Fig. 8) requires percolation, even with the calibrated $\mathrm{x}_{0}\left(\left|E_{\mathrm{c}}\right|\right)$ and $\mathrm{y}\left(\left|E_{\mathrm{c}}\right|\right)$ in Fig. 5. The turnaround can be otherwise only obtained by artificially reducing $\mathrm{Y}\left(\left|E_{\mathrm{c}}\right|\right)$ by $\sim 9 \times$. (b)(c): The decomposition of the simulated $\Delta V_{\mathrm{TH}}-V_{\mathrm{ov}}$ into (b) CT and (c) FE components. In (b), the $\Delta V_{\mathrm{TH}}{ }^{(\mathrm{CT})}-V_{\text {ov }}$ curves follow similar a power law with an exponent $<2$. In (c), a large exponent in $\Delta V_{\mathrm{TH}}{ }^{(\mathrm{FE})}-V_{\text {ov }}(>3.4)$ is required to capture the overall $\Delta V_{\mathrm{TH}}-V_{\mathrm{ov}}$ "turnaround" in (a).

phenomenon can be understood as a positive BTI (PBTI)dominated $V_{\mathrm{TH}}$ increase for lower $V_{\mathrm{ov}}(<1.2 \mathrm{~V})$ and a FE $V_{\mathrm{TH}}$ PGM-dominated $V_{\mathrm{TH}}$ descending thereafter. However, the turnaround behavior suggests that the $\Delta V_{\mathrm{TH}}$ contributions of charge trapping and of FE $V_{\mathrm{TH}}$ PGM should follow remarkably different $V_{\text {ov }}$ dependence (Fig. 9(b)(c)). Indeed, the success of the percolation model is that it introduces a very large exponent $(\sim 4.7)$ in the power-law dependence of $\Delta V_{\mathrm{TH}}{ }^{(\mathrm{FE})}$ on $V_{\mathrm{ov}}$ (Fig. $9(\mathrm{c}))$, such that it overturns the sluggish $\Delta V_{\mathrm{TH}}{ }^{(\mathrm{CT})}$ growth $\left(\sim V_{\mathrm{ov}}{ }^{2.0}\right.$, Fig. $\left.9(\mathrm{~b})\right)$. This is not possible without invoking percolation, even if by using the calibrated FE parameters in Table I (Fig. 9(a)). As a reference, an artificially constructed $f\left(E_{\mathrm{c}}{ }^{-}, E_{\mathrm{c}}{ }^{+}\right)$with $\sim 9 \times$ smaller $\left|E_{\mathrm{c}}\right|$ spread can qualitatively mimic the turnaround in a non-percolation manner (Fig. 9(a)), but this is way less plausible and hence not adopted.

It is worth noting that the fitted $\Delta V_{\mathrm{TH}}{ }^{(\mathrm{FE})}-V_{\mathrm{ov}}$ (Fig. 9(c)) does not imply $\mathrm{P}_{\mathrm{FE}}$ switching so nonlinearly versus $V_{\mathrm{ov}}$ in the case of percolation. In fact, the $\Delta \mathrm{P}_{\mathrm{FE}}$ during the $t_{\text {stress }}$ appears rather quasi-linear $\left(\sim V_{\mathrm{ov}}{ }^{1.6}\right.$, Fig. $\left.10(\mathrm{a})\right)$; rather, it is the arctan-form of the env $\left(\mathrm{P}_{\mathrm{FE}}\right)$ in Eqs. (5) that converts $\Delta \mathrm{P}_{\mathrm{FE}}$ nonlinearly into $\Delta V_{\mathrm{TH}}{ }^{(\mathrm{FE})}$ (Fig. 10(b)). In other words, the turnaround occurs because the $\mathrm{P}_{\mathrm{FE}}$ is approaching $\mathrm{P}_{\mathrm{c}}$ with increasing $V_{\mathrm{ov}}$, along which the chance of percolation and the resultant $\left|\Delta V_{\mathrm{TH}}{ }^{(\mathrm{FE})}\right|$ increase significantly; this eventually eclipses the PBTI-like $\Delta V_{\mathrm{TH}}{ }^{(\mathrm{CT})}$ (Fig. 9(b)) and turns the net $\Delta V_{\mathrm{TH}}$ into descending.

\section{2) Quasi-DC $I_{D, \text { lin }}-V_{G}$ measurements prediction}

We further use our FeFET CM to study the hysteretic and steep-slope behaviors in dual-trace $I_{\mathrm{D}, \operatorname{lin}}-V_{\mathrm{G}}$ quasi-DC measurements; they are swept from $-3 \mathrm{~V}$ to different maximum $V_{\mathrm{G}}\left(V_{\mathrm{G}, \max }\right)$ values (Fig. 11(a)). We argue that the SSFeFET "traits" can be interpreted under a unified physical ansatz, as is suggested by the steep slope observation on NVM-FeFETs [17]. Indeed, using the percolation model, we are able to reproduce both the hysteresis direction change with the $V_{\mathrm{G}, \max }$ and the local steep slope on the down-trace sweep (Fig. 11(b)).
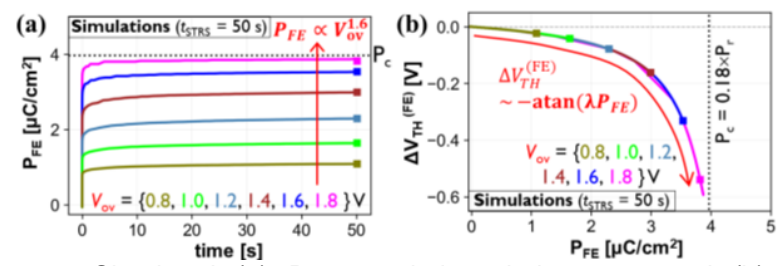

Fig. 10. Simulated (a) $\mathrm{P}_{\mathrm{FE}}-t$ evolution during $t_{\text {stress }}$ and (b) the associated $\Delta V_{\mathrm{TH}}{ }^{(\mathrm{FE})}-\mathrm{P}_{\mathrm{FE}}$ after the $t_{\mathrm{Stress}}$, for $V_{\mathrm{ov}}$ between 0.8 and $1.8 \mathrm{~V}$ and $t_{\text {stress }} \sim 50 \mathrm{~s}$ in Fig. 8. While $\Delta \mathrm{P}_{\mathrm{FE}}$ is only $\sim V_{\text {ov }}{ }^{1.6}$, the $\arctan$ form of $\Delta V_{\mathrm{TH}}{ }^{(\mathrm{FE})}-\mathrm{P}_{\mathrm{FE}}$ in Eqs. (5) is highly nonlinear and leads to the $\Delta V_{\mathrm{TH}}{ }^{(\mathrm{FE})}$ $\sim V_{\text {ov }}{ }^{4.7}$ in Fig. 9(c).

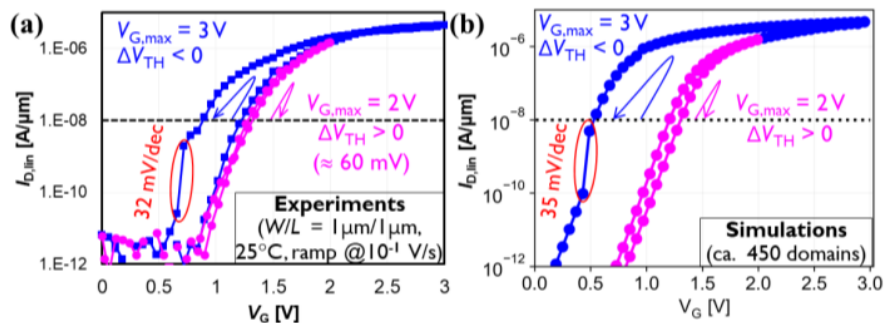

Fig. 11. Comparison of the (a) measured and (b) simulated $I_{D, \text { lin }}-V_{G}$ on $1 \mu \mathrm{m} / 1 \mu \mathrm{m}$ FeFETs. $V_{\text {TH }}$ is intercepted at $10 \mathrm{nA} \times W / L$. The local steep slope occurs during the down-sweep upon partial de-percolation of the positive domain "clusters" as $\mathrm{P}_{\mathrm{FE}}$ drops from $\mathrm{P}_{\mathrm{C}}$.

For one thing, the change of hysteresis from positive $\Delta V_{\mathrm{TH}}$ to negative is attributed to the turning point voltage $\left(V_{\mathrm{TP}}\right)$ that lies beyond $V_{\mathrm{G}, \max }=2 \mathrm{~V}$ (Fig. 12(a)). Hence sweeping to $2 \mathrm{~V}$ does not suffice to close in on $\mathrm{P}_{\mathrm{c}}$ (Fig. 12(b)) and ends up with a positive $\Delta V_{\mathrm{TH}}$, similar to that in eMSM (Fig. 10). Moreover, we can relate the steep $I_{\mathrm{D}}-V_{\mathrm{G}}$ slope on the down-sweep from $V_{\mathrm{G}, \max }$ $=3 \mathrm{~V}$ to "de-percolation" by analyzing the $V_{\mathrm{TH}}$ snapback on $\Delta V_{\mathrm{TH}}-V_{\mathrm{G}}$ (Fig. 12(a)). As $V_{\mathrm{G}}$ is swept down from $3 \mathrm{~V}, \mathrm{P}_{\mathrm{FE}}$ will also depart from $\mathrm{P}_{\mathrm{c}}$ (Fig. 12(b)), which will close the highly inverted conducting paths beneath the $+\mathrm{P}_{\mathrm{r}}$ domain clusters in a "branch-by-branch" fashion (Fig. 2(b)) and thus lead to successive $V_{\mathrm{TH}}$ snapbacks (Fig. 12(a)). It is the $V_{\mathrm{TH}}$ snapback in the subthreshold regime that eventually translates to the local steep slope on $I_{\mathrm{D}}-V_{\mathrm{G}}$.

Note that the interpretation of steep slope above implicitly assumes that the de-percolation process proceeds independently of the electric field, since the $\mathrm{P}_{\mathrm{FE}}$ depolarization is modeled by instant Preisach switching (Section II-B) and will hence continue regardless of the $V_{\mathrm{TH}}$ snapback it is creating. In Section IV, we will demonstrate with more refined domain reversal dynamics that such field-independence is a necessary condition for the $\Delta V_{\mathrm{TH}}$ snapback to manifest and consequently for the steep slope to be observed.

We have additionally studied the dimensional scaling aspect of the FeFET percolation model in our recent work [18]. This matters because changing $W / L$ will alter the shape of the lattice in percolation theory, making the $\mathrm{P}_{\mathrm{c}}$ value no longer conserve [27]. For example, $\mathrm{P}_{\mathrm{c}}$ will drop from $0.18 \mathrm{P}_{\mathrm{r}}$ to $0.06 \mathrm{P}_{\mathrm{r}}$ as $W / L$ changes from 1 to 2 and will further reduce at shorter $L$ [18]. In addition, for FeFETs with exceedingly large $W / L$, we can conveniently combine the percolation model with sub-FET parallelizing (Fig. 13(b)), where the transistor is sliced in width direction into multiple sub-FETs. This reduces the $W_{\text {sub }} / L$ of each sub-FET such that the associated "sub- $\mathrm{P}_{\mathrm{c}}$ " can be easily looked up (e.g., "sub- $\mathrm{P}_{\mathrm{c}}$ " $\approx 0.06 \mathrm{P}_{\mathrm{r}}$ for $W_{\text {sub }} / L=2$ ) without


Fig. 12. (a): Simulated on-the-fly $\Delta V_{T H}$ during the $I_{D, \text { lin }}-V_{G}$ sweeps in Fig. 11. $\Delta V_{T H}$ is referenced to the starting point of the sweeps $(-3 \mathrm{~V})$. The "turning point voltage" $V_{\mathrm{TP}}(=2.1 \mathrm{~V})$ defines the $V_{\mathrm{G}, \max }$ for $\Delta V_{\mathrm{TH}}-V_{\mathrm{G}}$ to turn around. The $V_{T H}$ snapback upon de-percolation occurs during the downsweep from $3 \mathrm{~V}$, which causes the steep slope on $I_{D, \text { lin }}-V_{G}(F i g .11(b))$. (b): The associated $\Delta V_{\mathrm{TH}}{ }^{(\mathrm{FE})}$ versus $\mathrm{P}_{\mathrm{FE}}$ simulated using $\operatorname{env}\left(\mathrm{P}_{\mathrm{FE}}\right)$. 




Fig. 13. (a) Comparison of the measured and simulated $I_{D, \text { lin }}-V_{G}$ sweeps for different transistor $W / L$ values up to $V_{\mathrm{G}, \max }=2 \mathrm{~V}$. The $1 \mu \mathrm{m} / 70 \mathrm{~nm}$ FeFET reaches $\Delta V_{T H}<0$ as contrary to the positive $\Delta V_{T H}$ in the $1 \mu \mathrm{m} / 1 \mu \mathrm{m}$ FeFET, and it shows local steep slope on the down-sweep. (b) Scheme of sub-FET parallelizing for simulating the $1 \mu \mathrm{m} / 70 \mathrm{~nm}$ FeFET. Each subFET assumes $W_{\text {sub }} / L=140 \mathrm{~nm} / 70 \mathrm{~nm}$ and has a "sub- $\mathrm{P}_{\mathrm{c}}$ " of $0.06 \mathrm{P}_{\mathrm{r}}$, which is smaller than the $\mathrm{P}_{\mathrm{c}}$ for $W / L=1 \mu \mathrm{m} / 1 \mu \mathrm{m}\left(\approx 0.18 \mathrm{P}_{\mathrm{r}}\right)$ and makes the percolation path formation more easily.

having to resort to the cumbersome $\mathrm{P}_{\mathrm{c}}$ extractions from MonteCarlo RN simulations [18]. Using this method, we can predict the experimentally observed negative $\Delta V_{\mathrm{TH}}$ in $1 \mu \mathrm{m} / 70 \mathrm{~nm}$ FeFETs when the $V_{\mathrm{G}, \max }$ is only $2 \mathrm{~V}$ (Fig. 13(a)). Compared to the $1 \mu \mathrm{m} / 1 \mu \mathrm{m}$ device (Fig. 11), the $1 \mu \mathrm{m} / 70 \mathrm{~nm}$ FeFET has a lower "sub- $\mathrm{P}_{\mathrm{c}}$ " $\left(\approx 0.06 \mathrm{P}_{\mathrm{r}}\right)$ in each constituting $140 \mathrm{~nm} / 70 \mathrm{~nm}$ subFET, which means easier percolation and hence a lower threshold $\mathrm{P}_{\mathrm{FE}}$ needed to "program" the $V_{\mathrm{TH}}$. The percolation in $1 \mu \mathrm{m} / 70 \mathrm{~nm}$ FeFETs also makes de-percolation possible and thus contributes to the steep slope during down-sweep (Fig. 13(a)).

\section{Nucleation-Growth Domain ReVersal Dynamics AND STEEP SLOPE}

So far, we have approximated the switching of individual FE domains by the instant Preisach model (Fig. 4), which does not include detailed domain reversal dynamics and hence is not able to cover the full picture of steep slope device physics. Meanwhile, although the commonly used nucleation-limited switching (NLS) model [11,12,24,31] does describe the domain reversal dynamics, it nevertheless misses the capability of reproducing the steep slope characteristics in SSFeFETs. This leads us to upgrading the instant Preisach switching model (Section II-B) to an NLS-derived, nucleation-growth (NG) domain reversal dynamics model, that phenomenologically explains the steep slope $I-V$ through the proposed fieldindependent domain growth [6]. Note that the charge trapping model and the channel percolation model remain unchanged.

We use the NLS scheme in [24] as the starting point for our NG model. The NLS model conceptualizes a slow, ratelimiting, field-dependent nucleation step and a subsequent fast, "unstoppable" domain growth step [31] (Fig. 14(a)). The nucleation barrier is characterized by a statistical distribution of

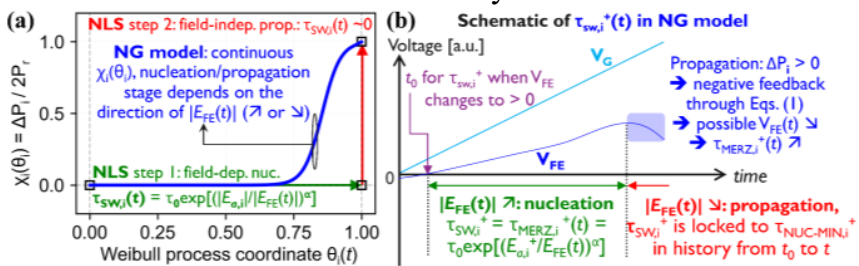

Fig. 14. (a) Comparison of the schematics (for domain $i$ ) of the two-step NLS model [31] and the continuous nucleation-growth (NG) model. The Weibull process coordinate $\theta_{\mathrm{i}}(t)$ follows [24]. (b) Schematic of $\mathrm{T}_{\mathrm{sw},{ }^{+}}{ }^{+}$(i.e., for $\Delta \mathrm{P}_{\mathrm{i}}>0$ ) calculation in the NG model for domain $i$. The $\mathrm{T}_{\mathrm{sw}, i^{+}}{ }^{+}$in growth step is phenomenologically locked to the minimum $\mathrm{T}_{\mathrm{sw}, \mathrm{i}^{+}}{ }^{+}$that has been set in the previous nucleation step $\left(\mathrm{T}_{\text {nuc-min, }}{ }^{+}\right)$. This guarantees that even if $V_{\mathrm{FE}}(t)$ decreases due to the negative feedback from $\Delta \mathrm{P}_{\mathrm{FE}}, \mathrm{T}_{\mathrm{sw}, i}{ }^{+}$will not increase but still respect $\mathrm{T}_{\text {nuc-min, } i^{+}}{ }^{+}$. The $\mathrm{T}_{\mathrm{sw}, \mathrm{i}}{ }^{-}$during $\Delta \mathrm{P}_{\mathrm{i}}<0$ can be similarly determined for $V_{\mathrm{G}}<0, V_{\mathrm{FE}}<0$.
TABLE II

EXTRACTEd HZO THIN FILM NuCLEATION DYNAMICS PARAMETERS [20]

\begin{tabular}{c|c|c}
\hline \hline Parameter & Median value & Spread \\
\hline Activation field $\left|E_{a}\right|^{\mathrm{a}}$ & $1.28 \mathrm{MV} / \mathrm{cm}$ & $0.64 \mathrm{MV} / \mathrm{cm}$ \\
Merz's law field dependence $\alpha$ & 3.602 & - \\
High-field switching time limit $\tau_{0}$ & $<<80 \mathrm{~ns}(<5 \mathrm{~ns})^{\mathrm{b}}$ & - \\
Weibull shape parameter $\beta$ & 1.014 & - \\
\hline \hline
\end{tabular}

${ }^{\mathrm{a}}\left|E_{a}\right|$ follows a Gaussian distribution [20].

${ }^{b} \mathrm{~T}_{0} \sim 80 \mathrm{~ns}$ (upper bound) was quantitatively fitted to our previous experiments [20], while our latest measurements have demonstrated a low-field $(\sim 4 \mathrm{MV} / \mathrm{cm})$ switching time $<5 \mathrm{~ns}$, meaning $\mathrm{T}_{0}<<5 \mathrm{~ns}$. This is confirmed by our in-house atomistic simulations ([32], $\mathrm{T}_{0} \sim 10^{-12} \mathrm{~s}$ ).

the activation field $E_{a}$ [24] resembling the $f\left(E_{\mathrm{c}}^{-}, E_{\mathrm{c}}^{+}\right)$in Fig. 4. However, we do not use the original NLS approximation suggested by [31], that a domain can flip up/down all together (Fig. 14(a)). This would misleadingly equate the "incipient" critical nuclei $\left(\sim 10^{0} \mathrm{~nm}^{3}[33]\right)$ to fully reversed domains $\left(>10^{1}\right.$ $\mathrm{nm}^{3}$ [29,33,34]). Rather, we believe that even for the non-ratelimiting "fast growth" step in NLS [31], we need to model it as a finite-rate process instead of an instant one. For this reason, we seek to "augment" the NLS model into our NG model.

The NG model is centered around a continuous function $\chi_{\mathrm{i}}\left(\theta_{\mathrm{i}}\right)$ (Fig. 14(a)) for each domain $i$, that smoothly combines the "conceptual" nucleation and growth steps in NLS [31]:

$$
\left\{\begin{array}{c}
\chi_{i}\left(\theta_{i}\right) \triangleq \Delta P_{i} /\left(2 P_{r}\right)= \pm\left[\tanh \left(\eta\left[\theta_{i}-H\right]\right)+1\right] / 2 \\
\theta_{i}=1-\exp \left\{-h_{i}{ }^{\beta}(t)\right\} \\
h_{i}(t)=\int_{t_{0}}^{t} 1 / \tau_{s w, i}(\tilde{t}) d \tilde{t}
\end{array}\right.
$$

Here, $\eta$ and $H$ are empirical parameters that can be adjusted to account for e.g., FE grain size refinement [36]. The "nucleation history parameter" $h_{i}(t)$, its exponent $\beta$ and the Weibull temporal process $\theta_{\mathrm{i}}(t)$ are formulated after [24]. The $t_{0}$ refers to the instant when $V_{\mathrm{FE}}$ turns positive (for polarizing from $-\mathrm{P}_{\mathrm{r}}$ to $+\mathrm{P}_{\mathrm{r}}$ ) or negative (for depolarizing from $+\mathrm{P}_{\mathrm{r}}$ to $-\mathrm{P}_{\mathrm{r}}$ ). $\tau_{\mathrm{sw}, \mathrm{i}}(t)$ is the domain-specific characteristic switching time and is related to the sampled activation field $E_{a, \mathrm{i}}$ (see Eqs. (7)).

To guarantee that nucleation should be the only rate-limiting step during domain reversal (Fig. 14(a)), we employ a phenomenological approach to determine $\tau_{\mathrm{sw}, \mathrm{i}}(t)$, based on both $E_{\mathrm{FE}}(t)\left(\right.$ or $\left.V_{\mathrm{FE}}(t)\right)$ and its direction (see Fig. 14(b)):

1. During nucleation, only "incipient" nuclei are formed, meaning $\Delta \mathrm{P}_{\mathrm{i}}$ is negligible and does not impose a noticeable negative feedback to $V_{\mathrm{FE}}$ through the " $V_{\mathrm{FE}, \mathrm{P}}$ " term in Eqs. (1). Hence $V_{\mathrm{FE}}$ will keep increasing with $V_{\mathrm{G}}$ according to " $V_{\mathrm{FE}, 0}$ " (Eqs. (1)), and $\tau_{\mathrm{sw}, \mathrm{i}}$ can safely fall back to the fielddependent expression $\tau_{\text {Merz,i }}$ in NLS per Merz's law [35].

2. During growth, significant polarization $\Delta \mathrm{P}_{\mathrm{i}}$ will be reversed, which would negatively feedback to $V_{\mathrm{FE}}$ through the " $V_{\mathrm{FE}, \mathrm{P}}$ " term in Eqs. (1), that can even lead $V_{\mathrm{FE}}$ to decreasing. This would in turn increase $\tau_{\text {Merz,i }}$ to be greater than the minimum $\tau_{\mathrm{sw}, \mathrm{i}}$ the domain has experienced during nucleation, thus contradicting the fundamental concept of "non-rate-limiting, fast growth step" [31]. To ensure that domain growth not overtake nucleation as the rate-limiting step, we phenomenologically lock $\tau_{\mathrm{sw}, \mathrm{i}}$ to its minimum value from the preceding nucleation step $\left(\tau_{\text {nuc-min,i }}\right)$. This essentially leads to a field-independent domain growth step, which proceeds with a $\tau_{\mathrm{sw}, \mathrm{i}}(t)$ that is always smaller than (or equal to) $\tau_{\text {nuc-min,i }}$ and disregards the larger $\tau_{\text {Merz,i }}(t)$ when $V_{\mathrm{FE}}(t)$ indeed snaps back. 

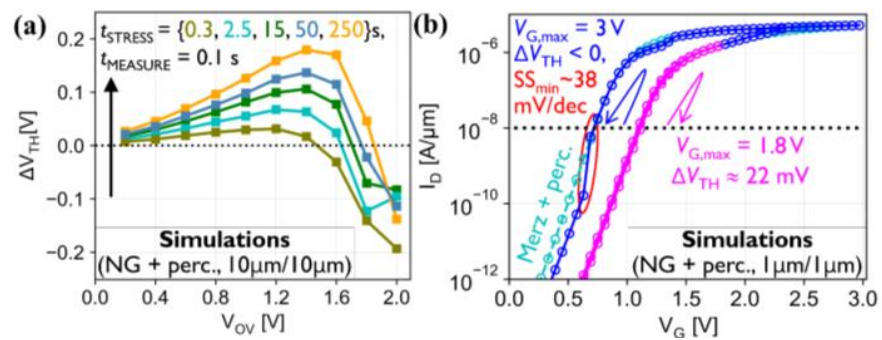

Fig. 15. Modeling prediction of (a) the eMSM (cf. Fig. 8) and the (b) $I_{D^{-}}$ $V_{G}$ sweeps (cf. Fig. 11), where the instant Preisach model is replaced by the NG dynamics model. In (a), the $\Delta V_{T H^{-}} V_{\text {ov }}$ turnaround in eMSM is successfully reproduced. For the $I_{D}-V_{G}$ prediction in (b), even with percolation, the down-sweep steep slope can only be reproduced with the field-independent domain growth in the NG model, but not with the misused, fully field-dependent Merz model even during growth.

Summarized mathematically, the NG model reads as follows:

$$
\left\{\begin{array}{c}
\tau_{M e r z, i}(t)=\tau_{0} \exp \left(\left|\frac{E_{a, i}}{V_{F E}(t) / t_{F E}}\right|^{\alpha}\right) \\
\tau_{s w, i}(t)=\min \left(\tau_{M e r z, i}(t), \tau_{n u c-\min , \mathrm{i}}\left(t_{0}, t\right)\right)
\end{array}\right.
$$

Here the $\tau_{\text {nuc-min,i }}\left(t_{0}, t\right)$ refers to the minimum $\tau_{\text {sw,i }}$ experienced from $t_{0}$ to $t$. The NLS parameters $\tau_{0}, \alpha, \beta$ and $E_{a}$ distribution in Eqs. (7) are extracted from temporal-voltage measurements on MFMCAPs ([20]; Table II). Because of the setup and devicerelated parasitic $\mathrm{RC}$ time constants $\left(\tau_{\mathrm{RC}}\right)$ in the measurement $(\sim$ $\left.10^{-9} \mathrm{~s}-10^{-6} \mathrm{~s}\right)[20]$, the extracted NLS parameters $\left(\tau_{0}, E_{a}\right.$, etc. $)$ can exhibit considerable discrepancies among literature $[11,24,33]$, depending upon the exact experimental setups. Nevertheless, the possible imperfection in the NLS parameters in Table II is not expected to compromise their use in interpreting our eMSM $\left(>10^{-2} \mathrm{~s}\right.$, Fig. 8(a)) or quasi-DC $I_{\mathrm{D}, \operatorname{lin}}-V_{\mathrm{G}}$ experiments $\left(\sim 10^{1} \mathrm{~s}\right.$, Fig. 11(a)). We argue this because these experiments were conducted at significantly "slower" time scales than $\tau_{\mathrm{RC}}$, where the NLS model fitting is essentially undisturbed by $\tau_{\mathrm{RC}}$ and can be safely applied to eMSM and $I_{\mathrm{D}, \mathrm{in}}-$ $V_{\mathrm{G}}$. In real-life digital circuits, the typical low-field $\left(\sim 10^{0}\right.$ $\mathrm{MV} / \mathrm{cm})$, room-temperature $(300 \mathrm{~K})$ switching time in (doped-)hafnia is believed to range $\sim 10^{0}$ ns [32], as is experimentally confirmed in [37] $(\sim 0.9 \mathrm{~ns}$ at $\sim 6 \mathrm{MV} / \mathrm{cm})$.

Replacing the instant Preisach model with the NG model, we can simultaneously reproduce (Fig. 15) the measured eMSM $\Delta V_{\mathrm{TH}^{-}} V_{\mathrm{ov}}$ in Fig. 8 and the dual-trace $I_{\mathrm{D}^{-}} V_{\mathrm{G}}$ sweeps for different $V_{\mathrm{G}, \max }$ in Fig. 11. Clearly, the simulated $\Delta V_{\mathrm{TH}}-V_{\mathrm{ov}}$ turnaround (Fig. 15(a)) confirms the effect of spatial percolation of of flipped-up regions in the FE layer on the channel conducting path formation (Fig. 2(b)). Following a parallel reasoning, the hysteresis direction change versus $V_{\mathrm{G}, \max }$ (Fig. 15(b)) can be also qualitatively reproduced.

Most importantly, the $I_{\mathrm{D}}-V_{\mathrm{G}}$ steep slope reproduction in Fig. 15(b) suggests that de-percolation alone does not suffice to engender the steep slope, if the field-independent domain growth is not included. This can happen if we wrongly apply the field-dependent Merz's law throughout the domain reversal including the growth step. In this case, even with the help of env $\left(\mathrm{P}_{\mathrm{FE}}\right)$, the model cannot reproduce the steep slope (Fig. 15(b)). Indeed, as shown in Fig. 12, it is the $V_{\mathrm{TH}}$ snapback in the subthreshold regime that leads to the local steep slope during $I_{\mathrm{D}, \text { lin }}-V_{\mathrm{G}}$ down-sweep. This would not be allowed (Fig. 16(a)) if we mistakenly apply the field-dependent Merz's law to both nucleation and growth, because the negative-feedback field dependence of $\tau_{\text {Merz,i }}$ will then adjust the depolarization
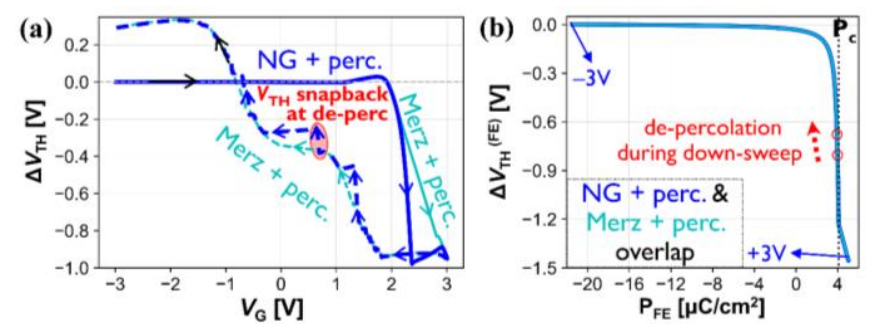

Fig. 16. Simulated (a) $\Delta V_{\mathrm{TH}}-V_{\mathrm{G}}$ and (b) $\Delta V_{\mathrm{TH}}{ }^{(\mathrm{FE})}-\mathrm{P}_{\mathrm{FE}}$ during the $I_{\mathrm{D}, \text { lin }}-V_{\mathrm{G}}$ sweeps as in Fig. 12, with the instant Preisach model replaced by the NG dynamics model. The de-percolation during down-sweep can only cause the $V_{T H}$ snapback if the field-independent domain growth is present in the NG model. This is not possible if we wrongly apply the field-dependent Merz's law to the entire domain switching process, even if we allow it to follow the same env $\left(\mathrm{P}_{\mathrm{FE}}\right)$-based $\Delta V_{\mathrm{TH}}{ }^{(\mathrm{FE})} \mathrm{P}_{\mathrm{FE}}$ in (b).

switching rate at which $\mathrm{P}_{\mathrm{FE}}$ departs from $\mathrm{P}_{\mathrm{c}}$ during depercolation (Fig. 16(b)), such that any potential $V_{\mathrm{TH}}$ snapback will be automatically smeared out. In fact, the $V_{\mathrm{TH}}$ snapbacks appear in Fig. 12 exactly due to the crude field-independent "dynamics" in the instant Preisach model $\left(\tau_{\mathrm{sw}, \mathrm{i}} \equiv 0\right)$. Therefore, in order for the steep slope to appear during de-percolation, it is essential that the minimum characteristic switching time set by the rate-limiting domain nucleation step (i.e., $\tau_{\text {nuc-min,i }}$ ) be faithfully upheld during the subsequent domain growth. In other words, it is the field-independent domain growth that governs the de-percolation profile along the $V_{\mathrm{G}}$-axis, which will eventually determine the $V_{\mathrm{TH}}$ snapback effect that is needed for the steep slope behavior.

\section{CONCLUSIONS}

This work presents a physics-based FeFET compact model that builds on a transient, self-consistent solution of the FEMOS electrostatics with a discretized ferroelectric polarization switching scheme. The model addresses three involved physical aspects that are crucial to the electrical interpretation of FeFETs: 1) the nonradiative multi-phonon charge trapping, 2) the channel percolation effect from the spatial configuration of FE domains and 3) the phenomenological nucleation-growth domain reversal dynamics. Based on a comprehensive hardware validation, we conclude that

1. The onset of source-to-drain FE domain percolation determines the channel conducting path formation that is needed to electrically sense the $V_{\mathrm{TH}}$ change, thus defining the gate bias required to initiate the programming of the FeFET $V_{\mathrm{TH}}$. Consequently, there is a channel geometry factor acting on top of the polarization charge in modulating the drain current.

2. Field-independent domain growth is the fundamental origin of the steep subthreshold slope. It operates in the course of channel "de-percolation" through unchecked FE domain depolarization, which ultimately causes $V_{\mathrm{TH}}$ to snapback in subthreshold region and steepens the $I_{\mathrm{D}^{-}} V_{\mathrm{G}}$.

3. The NLS-derived, nucleation-growth interpretation of the steep slope $I-V$, although being phenomenological, provides an alternative modeling approach of SSFeFETs, thus opening up the possibility of establishing a unified understanding of NVM-FeFETs and logic-oriented SSFeFETs. Further investigation of domain reversal dynamics is needed for refining the field-independent domain growth formalism. 


\section{ACKNOWLEDGMENT}

The authors gratefully acknowledge M. Thesberg (TU Wien), Ph. Roussel, A. S. Verhulst, B. Truijen, B. O'Sullivan, U. Celano and S. Clima for the fruitful discussions.

\section{REFERENCES}

[1] J. Müller et al., "Ferroelectric Zr0.5Hf0.5O2 thin films for nonvolatile memory applications", Appl. Phys. Lett., 99, 2011, art. 112901, doi:10.1063/1.3636417.

[2] M. Trentzsch et al., "A 28nm HKMG super low power embedded NVM technology based on ferroelectric FETs", 2016 IEEE International Electron Devices Meeting (IEDM), San Francisco, CA, USA, 2016, pp. 11.5.1-11.5.4, doi:10.1109/IEDM.2016.7838397.

[3] K. Florent et al., "Vertical Ferroelectric $\mathrm{HfO}_{2}$ FET based on 3-D NAND Architecture: Towards Dense Low-Power Memory", 2018 IEEE International Electron Devices Meeting (IEDM), San Francisco, CA, USA, 2018, pp. 2.5.1-2.5.4, doi:10.1109/IEDM.2018.8614710.

[4] Z. Krivokapic et al., "14 nm Ferroelectric FinFET Technology with Steep Subthreshold Slope for Ultra Low Power Applications", 2017 IEEE International Electron Devices Meeting (IEDM), San Francisco, CA, USA, 2017, pp. 15.1.1-15.1.4, doi:10.1109/IEDM.2017.8268393.

[5] M. N. K. Alam et al., "On the Characterization and Separation of Trapping and Ferroelectric Behavior in HfZrO FET," in IEEE J. Elec. Dev. Soc., vol. 7, pp. 855-862, 2019, doi:10.1109/JEDS.2019.2902953.

[6] J. Van Houdt and P. Roussel, "Physical Model for the Steep Subthreshold Slope in Ferroelectric FETs", IEEE Elec. Dev. Lett., vol. 39, no. 6, pp. 877-880, 2018, doi:10.1109/LED.2018.2829604.

[7] M. N. K. Alam, P. Roussel, M. Heyns and J. Van Houdt, "Positive nonlinear capacitance: the origin of the steep subthreshold-slope in ferroelectric FETs", Scientific Reports, 9, 2019, art. 14957, doi:10.1038/s41598-019-51237-2.

[8] C. Jin, T. Saraya, T. Hiramoto and M. Kobayashi, "Physical Mechanisms of Reverse DIBL and NDR in FeFETs With Steep Subthreshold Swing", IEEE J. Elec. Dev. Soc., vol. 8, pp. 429-434, 2020, doi:10.1109/JEDS.2020.2986345.

[9] B. Obradovic, T. Rakshit, R. Hatcher, J. A. Kittl and M. S. Rodder, "Ferroelectric Switching Delay as Cause of Negative Capacitance and the Implications to NCFETs," 2018 Symposium on VLSI Technology, Honolulu, HI, 2018, pp. 51-52, doi:10.1109/VLSIT.2018.8510628.

[10] T. Mikolajick, U. Schroeder and S. Slesazeck, "The Past, the Present, and the Future of Ferroelectric Memories", IEEE Trans. Elec. Dev., vol. 67, no. 4, pp. 1434-1443, doi:10.1109/TED.2020.2976148.

[11] K. Ni, W. Chakraborty, J. Smith, B. Grisafe and S. Datta, "Fundamental Understanding and Control of Device-to-Device Variation in Deeply Scaled Ferroelectric FETs," 2019 Symposium on VLSI Technology, Kyoto, Japan, 2019, pp. T40-T41, doi:10.23919/VLSIT.2019.8776497.

[12] S. Deng et al., "A Comprehensive Model for Ferroelectric FET Capturing the Key Behaviors: Scalability, Variation, Stochasticity, and Accumulation", Proc. Symposium on VLSI Technology, 2020, pp. TF1.4.1- TF1.4.2.

[13] Y. Higashi et al., "Impact of Charge trapping on Imprint and its Recovery in $\mathrm{HfO}_{2}$ based FeFET," 2019 IEEE International Electron Devices Meeting (IEDM), San Francisco, CA, USA, 2019, pp. 15.6.1-15.6.4, doi:10.1109/IEDM19573.2019.8993472.

[14] I. Stolichnov et al., "Genuinely Ferroelectric Sub-1-Volt-Switchable Nanodomains in $\mathrm{Hf}_{\mathrm{x}} \mathrm{Zr}_{(1-\mathrm{x})} \mathrm{O}_{2}$ Ultrathin Capacitors", ACS Appl. Mater. Interfaces, 10, pp. 30514-30521, 2018, doi:10.1021/acsami.8b07988.

[15] U. Celano et al., "Ferroelectricity in Si-Doped Hafnia: Probing Challenges in Absence of Screening Charges", Nanomaterials, 10(8), 1576, 2020, doi:10.3390/nano10081576.

[16] B. Kaczer et al., "The Relevance of Deeply-Scaled FET Threshold Voltage Shifts for Operation Lifetimes", 2012 IEEE International Reliability Physics Symposium (IRPS), Anaheim, CA, 2012, pp. 5A.2.15A.2.6, doi:10.1109/IRPS.2012.6241839.

[17] H. Mulaosmanovic et al., "Impact of Read Operation on the Performance of $\mathrm{HfO}_{2}$-Based Ferroelectric FETs", IEEE Elec. Dev. Lett., vol. 41, no. 9, pp. 1420-1423, 2020, doi:10.1109/LED.2020.3007220.

[18] Y. Xiang et al., "Implication of Channel Percolation in Ferroelectric FETs for Threshold Voltage Shift Modeling", 2020 IEEE International Electron Devices Meeting (IEDM), San Francisco, CA, USA, 2020, pp. 18.2.1-18.2.4

[19] Y. Xiang et al., "Physical Insights on Steep Slope FEFETs including
Nucleation-Propagation and Charge Trapping", 2019 IEEE International Electron Devices Meeting (IEDM), San Francisco, CA, USA, 2019, pp. 21.6.1-21.6.4, doi:10.1109/IEDM19573.2019.8993492.

[20] M. N. K. Alam et al., "HfZrO Ferroelectric Characterization and Parameterization of Response to Arbitrary Excitation Waveform", Proc. IEEE SOI-3D-Subthreshold Microelectronics Technology Unified Conference (S3S), San Jose, CA, USA, 2019, art. 3.01.

[21] G. Rzepa et al., "Comphy - A compact-physics framework for unified modeling of BTI", Microelectronics Reliability, vol. 85, pp. 49-65, 2018, doi:10.1016/j.microrel.2018.04.002.

[22] H. Agarwal et al., "BSIM-BULK106.2.0 MOSFET Compact Model: Technical Manual", Univ. California, Berkeley, 2017, bsim.berkeley.edu/models/bsimbulk/.

[23] M. A. Wahab and M. A. Alam, "A Verilog-A Compact Model for Negative Capacitance FET" (version 1.1.3), nanoHUB, doi:10.4231/D3QZ22K3Z.

[24] C. Alessandri, P. Pandey and A. C. Seabaugh, "Experimentally Validated, Predictive Monte Carlo Modeling of Ferroelectric Dynamics and Variability," 2018 IEEE International Electron Devices Meeting (IEDM), San Francisco, CA, 2018, pp. 16.2.1-16.2.4, doi:10.1109/IEDM.2018.8614607.

[25] M. Pešić et al., "Physical Mechanisms behind the Field-Cycling Behavior of $\mathrm{HfO}_{2}$-Based Ferroelectric Capacitors", Adv. Func. Mater., 26, pp. 4601-4612, 2016, doi:10.1002/adfm.201600590.

[26] S. Kirkpatrick, "Percolation and Conduction", Rev. Mod. Phys., vol. 45, no. 4, pp. 574-588, 1973, doi:10.1103/RevModPhys.45.574.

[27] D. Stauffer, "Scaling theory of percolation clusters", Phys. Rep., vol. 54, 1, pp. 1-74, 1979, doi:10.1016/0370-1573(79)90060-7.

[28] B. J. Last and D. J. Thouless, "Percolation Theory and Electrical Conductivity", Phys. Rev. Lett., vol. 27, no. 25, pp. 1719-1721, 1971, doi:10.1103/PhysRevLett.27.1719.

[29] H. Mulaosmanovic, T. Mikolajick and S. Slesazeck. "Accumulative Polarization Reversal in Nanoscale Ferroelectric Transistors", ACS Appl. Mater. Interfaces, 10, pp. 23997-24002, 2018, doi:10.1021/acsami.8b08967.

[30] B. Kaczer et al., "Ubiquitous relaxation in BTI stressing-New evaluation and insights," 2008 IEEE International Reliability Physics Symposium (IRPS), Phoenix, AZ, 2008, pp. 20-27, doi:10.1109/RELPHY.2008.4558858.

[31] A. K. Tagantsev, I. Stolichnov, N. Setter, J. S. Cross, and M. Tsukada, "Non-Kolmogorov-Avrami switching kinetics in ferroelectric thin films", Phys. Rev. B, 66, 2002, art. 214109, doi:10.1103/PhysRevB.66.214109.

[32] S. Clima et al., "Ferroelectric Switching in FEFET: Physics of the Atomic Mechanism and Switching Dynamics in $\mathrm{HfZrO}_{x}, \mathrm{HfO}_{2}$ with Oxygen Vacancies and Si dopants", 2020 IEEE International Electron Devices Meeting (IEDM), San Francisco, CA, USA, 2020, pp. 4.2.1-4.2.4.

[33] K. Lee et al., "Stable Subloop Behavior in Ferroelectric Si-doped $\mathrm{HfO}_{2}$ ", ACS Appl. Mater. Interfaces, 11, pp. 38929-38936, 2019, doi: 10.1021/acsami.9b12878.

[34] A. K. Saha, K. Ni, S. Dutta, S. Datta and S. Gupta, "Phase field modeling of domain dynamics and polarization accumulation in ferroelectric HZO", Appl. Phys. Lett., 114, 2019, art. 202903, doi:10.1063/1.5092707.

[35] W. J. Merz, "Domain Formation and Domain Wall Motions in Ferroelectric $\mathrm{BaTiO}_{3}$ Single Crystals", Phys. Rev., vol. 95, no. 3, pp. 690698, 1954, doi:10.1103/PhysRev.95.690.

[36] H. K. Yoo et al., "Engineering of Ferroelectric Switching Speed in $\mathrm{Si}$ Doped $\mathrm{HfO}_{2}$ for High-Speed 1T-FERAM Application", 2017 IEEE International Electron Devices Meeting (IEDM), San Francisco, CA, USA, 2017, pp. 19.6.1-19.6.4, doi: 10.1109/IEDM.2017.8268424.

[37] X. Lyu, M. Si, P. R. Shrestha, K. P. Cheung and P. D. Ye, "First Direct Measurement of Sub-Nanosecond Polarization Switching in Ferroelectric Hafnium Zirconium Oxide", 2019 IEEE International Electron Devices Meeting (IEDM), San Francisco, CA, USA, 2019, pp. 15.2.1-15.2.4, doi: 10.1109/IEDM19573.2019.8993509. 